\title{
Young Stars and Planets Near the Sun: Explosive Phenomena from Falling Evaporating Bodies
}

\author{
Subhon Ibadov ${ }^{1,2}$ and Firuz S. Ibodov ${ }^{1}$ \\ ${ }^{1}$ Lomonosov Moscow State University, Sternberg Astronomical Institute, 119991 Moscow, \\ Russia \\ email: mshtf@sai.msu.ru \\ ${ }^{2}$ Institute of Astrophysics, Tajik Academy of Sciences, 734042 Dushanbe, Tajikistan \\ email: ibadovsu@yandex.ru
}

\begin{abstract}
Impacts of falling evaporating bodies (FEBs) with stars and planets at velocities $V \gtrsim 10-20 \mathrm{~km} / \mathrm{s}$ will be accompanied, due to aerodynamic effects such as crushing and transversal expansion of the crushed mass, by the FEB's "explosion" and the generation of a strong "blast" wave, resulting in FEB-generated explosive/flare phenomena. Multiwavelength monitoring of nearby young stars (and exoplanets) with dense protoplanetary disks rich in FEB's is hence of interest for identifying such FEB-related mechanisms possibly underlying their variability.
\end{abstract}

Keywords. Young stars, falling evaporating bodies, FEB-generated explosive/flare phenomena

\section{Introduction}

Spectral observations of nearby, young stars like $\beta$ Pictoris indicate the presence of fluxes of comet-like falling evaporating bodies (FEBs; e.g., Lagrange et al. 1987). More than 2000 sungrazing comets (perihelion distances $\lesssim 0.01 \mathrm{AU}$ ) were discovered during the last three decades (Weissman 1983†). Fast thermalization of the kinetic energy of these bodies leads to high-temperature explosive phenomena (cf. Ibadov 1990; 2011; Ibadov et al. 2009). One such giant meteor observed was the June 30, 1908 Tunguska fireball, which exploded at heights range $5-10 \mathrm{~km}$ with an energy release of about 20 megatons of TNT.

An analytic approach to the explosion of bolides in planetary atmospheres was developed by Prof. Grigorian during 1976-1979 by modifying the basic equations of the physical theory of meteors. This approach was further developed in connection with the 100th anniversary of the Tunguska phenomenon. The complete analytic theory of bolide explosions was presented and used to explain the February 15, 2013 Chelyabinsk event (Grigorian et al. 2013 and references therein).

Fortunately, Tunguska-like planetary explosive phenomena in our Solar System have small probabilities, i.e., about one event per 200-300 years. Thus, young stars (and exoplanets) located close to the Sun, for which FEB explosive phenomena should be far more frequent, are unique natural laboratories to study the early evolution of the Sun/stars and planets, including their habitability. Here, we present some theoretical results concerning evolution of FEBs in the atmospheres of young stars and planets, derived on the basis of the above-mentioned theory.

$\dagger$ See also http://sungrazer.nrl.navy.mil 


\section{Explosions of FEBs in Stellar and Planetary Atmospheres}

Calculations show that intense aerodynamic crushing of FEBs along with transversal expansion occurs within the stellar/solar chromosphere. Disintegration of FEBs within the chromosphere has a meteor-like character and can cause anomalously high abundances of atoms of refractory elements, such as Fe and Si. Moreover, additional emission due to such metal atoms can be detected above the background of solar coronal radiation, if the FEBs' radii are $\gtrsim 100 \mathrm{~m}$ (cf. Ibadov et al. 1999).

It should be noted that comet nuclei/FEBs can also be completely destroyed within the solar corona, but that destruction may be due to another processes, such as collisions with other circumsolar bodies. Comet outbursts are potential observational manifestations of such phenomena (cf. Schrijver et al. 2012; Ibadov 2012 and references therein).

The equation for the kinetic energy loss of aerodynamically fully crushed and flattening FEBs allows the determination of all parameters related to the place of maximum energy release (due to FEB explosion) in an atmosphere (Grigorian et al. 2013). The characteristic time for thermalization of the FEB's kinetic energy within the "exploding" layer is $\sim 0.3 \mathrm{sec}$, at a layer thickness of roughly $0.7 H=140 \mathrm{~km}$ (where $H$ is height scale of photosphere). FEBs similar to comet Halley 1986 III would release explosive energy close to the energies of large solar flares, $10^{32} \mathrm{erg}$, while FEBs similar to comet Hale-Bopp 1995 OI (radius $\gtrsim 30 \mathrm{~km}$ ) should lead to stellar super-flares (Grigorian et al. 2000; Ibadov et al. 2009; Eichler \& Mordecai 2012). Using similar arguments, we have explained the heights of plumes observed with HST during the collision of comet SL-9 with Jupiter in July 1994 and have predicted the ejection of plumes from young stars due to FEBs (Ibodov \& Ibadov 2014 and references therein).

\section{Conclusions}

Nearby young stars and planets are unique objects for understanding the evolution of our Solar System during the epoch of intense impacts with FEBs. Impacts of FEBs should produce hot $\left(\sim 10^{7} \mathrm{~K}\right)$ plasma, strong shock waves, plasma plumes, and anomalously high abundances of metal atoms. Multi-wavelength observations/monitoring of nearby young stars and exoplanets are of interest for studying/identifying such potential mechanisms underlying their flare activity (e.g., Teets et al. 2011).

\section{Acknowledgements}

The authors are grateful to Prof. J. H. Kastner and Dr. S. Lepine for their invitation to IAUS 314, SAI MSU for hospitality, the editor for constructive comments, and E. Shimanovskaya and N. Gostev for technical support.

\section{References}

Eichler, D. \& Mordecai, D. 2012, ApJL, 761, L27

Grigorian, S. S., Ibadov, S., \& Ibodov, F. S. 2000, D-Ph, 45, 463

Grigorian, S. S., Ibodov, F. S., \& Ibadov, S. I. 2013, Sol. Sys. Res., 47, 268

Ibadov, S. 1990, Icarus, 86, 283

Ibadov, S. 2011, in Proc.IAUS 283, A.Manchado, L.Stanghellini, \& D.Schonberner, eds., p.392

Ibadov, S. 2012, Adv.Space Res., 49, 467

Ibadov, S, Ibodov, F. S. \& Grigorian, S. S. 1999, Rom. As. J Suppl, 9, 125

Ibadov, S. et al. 2009, in Proc. IAUS 25\%, N. Gopalswamy \& D. F. Webb, eds., p. 341

Ibodov, F. S. \& Ibadov, S. 2014, in Proc. IAUS 300, B. Schmieder et al., eds., p. 509.

Lagrange, A. M., Ferlet, R., \& Vidal-Madjar, A. 1987, A\&A, 173, 289

Schrijver, C. J., Brown, J. C., Battams, K., et al. 2012, Science, 335, 324

Teets, W. K., Weintraub, D. A., Grosso, N., et al. 2011, ApJ, 741, 83

Weissman, P. R. 1983, Icarus, 55, 448 